\title{
Um caso de diabetes de difícil controlo
}

Clara Jasmins, * Ana Rita Mendes,* Gonçalo Melo*

\section{RESUMO}

Introdução: A diabetes autoimune latente do adulto caracteriza-se pelo aparecimento de diabetes mellitus em indivíduos com menos de 50 anos, com quadro inicial de polifagia, polidipsia e/ou poliúria, IMC $<25 \mathrm{~kg} / \mathrm{m} 2$ e história pessoal ou familiar de doenças autoimunes.

Descrição do caso: Doente de 46 anos, sexo masculino, desempregado, com antecedentes pessoais de défice cognitivo, abuso de substâncias tóxicas e perturbações depressivas e de ansiedade. Como antecedente familiar refere que o pai foi diagnosticado com diabetes mellitus aos 40 anos. Nas suas relações familiares apresenta alguns conflitos com a irmã. Assintomático até outubro de 2012, quando recorre à consulta por cansaço, xerostomia, polifagia, polidipsia, poliúria e perda ponderal acentuada, com novo consumo abusivo de álcool, tabaco e comportamentos desadequados. Coloca-se a hipótese diagnóstica de diabetes mellitus inaugural, apresentando HbA1c $12 \%$ e glicemia em jejum $254 \mathrm{mg} / \mathrm{dL}$. Inicia terapêutica com metformina e nateglinida. Porém, mantém mau controlo da doença, com necessidade de escalada terapêutica sucessiva, até introdução de insulina dois meses após o diagnóstico. É referenciado à consulta de diabetologia e falta por diversas vezes às consultas de seguimento, até ao diagnóstico de diabetes autoimune latente do adulto (LADA) em fevereiro de 2015, quando apresenta anticorpos anti-GAD65 positivos. Por insuficiência económica extrema são várias as refeições que nesta altura não consegue cumprir e apresenta vários episódios de hipoglicemia e queixas de cansaço e irritabilidade, ainda que sem alteração do estado de consciência. Em janeiro de 2016 é internado por quadro de cetoacidose diabética. Desde então tem mais apoio social, sem novos episódios de hipoglicemia. Contudo, mantém níveis inadequados de HbA1c.

Comentário: O caso clínico que se descreve mostra a influência das condições socioeconómicas, antecedentes pessoais e as relações familiares no tratamento de doenças crónicas como a LADA. Os hábitos alimentares desadequados, com refeições escassas e em períodos irregulares, além de má adesão aos cuidados de saúde e à terapêutica, condicionaram um controlo ineficaz da doença. Pretende-se com este caso salientar a importância de capacitar o doente pelo seu próprio tratamento e promover a participação ativa dos familiares mais próximos. Neste caso em particular, a relação conflituosa do doente com a irmã constituiu mais um elemento perturbador para o sucesso terapêutico.

Palavras-chave: Diabetes autoimune; Dieta; Fatores socioeconómicos.

\section{INTRODUÇÃO}

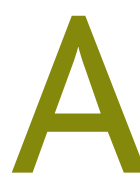

diabetes autoimune latente do adulto caracteriza-se por um aparecimento em indivíduos na quarta e quinta décadas de vida e não-obesos. ${ }^{1-2} \mathrm{O}$ IMC destes doentes é por norma mais elevado que o daqueles com diabetes mellitus (DM) tipo 1, mas inferior ao dos doentes com DM tipo 2.

A nível global, entre 4 a 14\% dos doentes diagnosticados com DM tipo 2 apresentam LADA. No norte da Europa, cerca de 7 a $14 \%$ apresentam anticorpos anti-GAD positivos, sendo que no Sudeste Europeu, Ásia e América do Norte, a prevalência é de 4 a $6 \%{ }^{8}$
Inicialmente apresentam uma boa resposta a antidiabéticos orais; porém, com necessidade gradual de introdução de insulina na terapêutica, normalmente após um período de pelo menos seis meses após o diagnóstico. Esta condição permite obter uma distinção entre a LADA e a DM tipo 1 tardia. ${ }^{1-3}$ Comparativamente aos doentes com DM tipo 2, as características mais frequentes são o aparecimento da doença em idade inferior a 50 anos, sintomas agudos ao início, IMC $<25 \mathrm{~kg} / \mathrm{m}^{2}$ e história pessoal ou familiar de doenças autoimunes. A presença de dois ou mais destes critérios apresentam uma sensibilidade de $90 \%$ e especificidade de $71 \%$ para o diagnóstico de LADA. ${ }^{3}$

Adicionalmente estes doentes apresentam níveis 
baixos de péptido C e anticorpos anti-GAD e anti-ICA positivos, pelo que a deteção dos últimos pode servir como um bom teste preditivo acerca da possível necessidade de introduzir insulina na terapêutica. ${ }^{1,4-5}$

Ressalve-se que nos doentes com anticorpos positivos, os níveis de péptido $C$ podem tornar-se indetetáveis ao fim de doze anos e que, apesar de não ser a condição mais frequente, a presença de obesidade per si não exclui o diagnóstico de LADA..$^{6-7}$

Descreve-se um caso clínico desta entidade rara, embora muitas vezes subdiagnosticada e facilmente confundível com outras patologias.

O objetivo deste relato de caso é melhorar o conhecimento acerca desta entidade, relembrando a marcha diagnóstica e salientando os possíveis fatores que podem levar a confundir má adesão à terapêutica com insuficiência terapêutica per si, relembrando que é possível que as duas situações ocorram no mesmo utente.

\section{DESCRIÇÃO DO CASO}

Doente de 46 anos, sexo masculino, desempregado, membro de família unitária, classe V na escala de Graffar, com antecedentes pessoais de défice cognitivo secundário, meningite na $1^{\mathrm{a}}$ infância e traumatismo crânio-encefálico aos seis anos, abuso crónico de álcool no passado (até dezembro de 2007), abuso do tabaco, perturbação depressiva com ideação suicida e ansiedade com impulsividade e défice de controlo de impulsos. Relativamente aos antecedentes familiares salienta-se o pai com diagnóstico de DM desde os 40 anos de idade. Tem ainda uma irmã da qual se desconhecem doenças, mas com quem mantém uma relação de proximidade, ainda que, por vezes, conflituosa.

Aparentemente assintomático até outubro de 2012, altura em que recorre à consulta por um quadro de cansaço, xerostomia, polifagia, polidipsia, poliúria e perda ponderal de seis quilogramas num mês, com novo consumo abusivo de álcool, tabaco e comportamentos desadequados. Ao exame objetivo apresenta-se agitado, sem outras alterações a registar. Nesta altura coloca-se como hipótese diagnóstica um quadro inaugural de DM, pelo que se pede uma avaliação laboratorial dirigida, que resulta em HbAlc $12 \%$ e glicemia em jejum $254 \mathrm{mg} / \mathrm{dL}$. Nesta altura é medicado com metformina e nateglinida $60 \mathrm{mg}$, após as duas únicas refeições que faz por dia de forma errática (por dificuldade econó- mica extrema). Por novo descontrolo da DM, ainda que sob terapêutica (glicemia pós-prandial $470 \mathrm{mg} / \mathrm{dL}$ ), em dezembro de 2012 suspende nateglinida e inicia insulina glargina $10 \mathrm{U}$ ao deitar. Apesar de tudo, esta dose é insuficiente e apresenta necessidade sucessiva de aumento de dose, perfazendo $18 \mathrm{U}$ ao deitar, em fevereiro de 2013. No mês seguinte, mantendo registos de glicemia capilar entre 200 e $300 \mathrm{mg} / \mathrm{dL}$ em jejum, aumenta a dose de insulina glargina para $22 \mathrm{U}$ ao deitar e inicia insulina humana regular $10 \mathrm{U}$ às refeições.

Em abril de 2013, por descontrolo total da glicemia e sistemática insuficiência terapêutica, põe-se a hipótese de DM tipo 1 ou LADA, pelo que fica a aguardar confirmação diagnóstica e é referenciado à consulta de diabetologia da Associação Protetora dos Diabéticos de Portugal (APDP). A reavaliação seguinte ocorre em julho de 2013, quando se aumenta de novo a insulina glargina para 26U/dia. Durante nove meses, o utente abandona a consulta e regressa em abril de 2014. Nessa altura suspende insulina glargina e inicia NPH intermédia $23 \mathrm{U}$ antes do pequeno-almoço e $13 \mathrm{U}$ antes do jantar. Sem sucesso quanto ao controlo da diabetes, pede-se um diário de ingestão alimentar e de glicemias capilares durante uma semana, que mostra valores de glicemias capilares muito irregulares, sobretudo em períodos de jejum prolongado. (Relembre-se, mais uma vez, que o doente tem insuficiência económica grave, dependendo da Santa Casa para ser alimentado, pelo que os horários não são estritamente cumpridos, além de que é uma alimentação muito rica em hidratos de carbono). Em maio de 2014 apresenta uma HbAlc de 9,8\%.

Em fevereiro de 2015 é confirmado o diagnóstico de diabetes latente autoimune do adulto (LADA) por anticorpos anti-GAD65 positivos e anticorpos anti-ilhéus pancreáticos em zona cinzenta. Porém, mantém erros alimentares, com dieta constituída maioritariamente por hidratos de carbono. Passa a fazer insulina NPH $20 \mathrm{U}$ ao pequeno-almoço e jantar. Dois meses depois recorre à consulta com queixas de maior irritabilidade e cansaço, sem alteração do estado de consciência. Nos registos de glicemia capilar apresenta alguns valores abaixo de $70 \mathrm{mg} / \mathrm{dL}$ em jejum, pelo que se reduz $2 \mathrm{U}$ de insulina à noite. Em maio de 2015 realiza novo ajuste da terapêutica: para metformina, inicia insulina mistura 25/75 $10 \mathrm{U}$ ao pequeno-almoço, $22 \mathrm{U}$ ao almoço e $24 \mathrm{U}$ ao jantar. Planeia-se dieta com pão ao levantar e sopa no lan- 


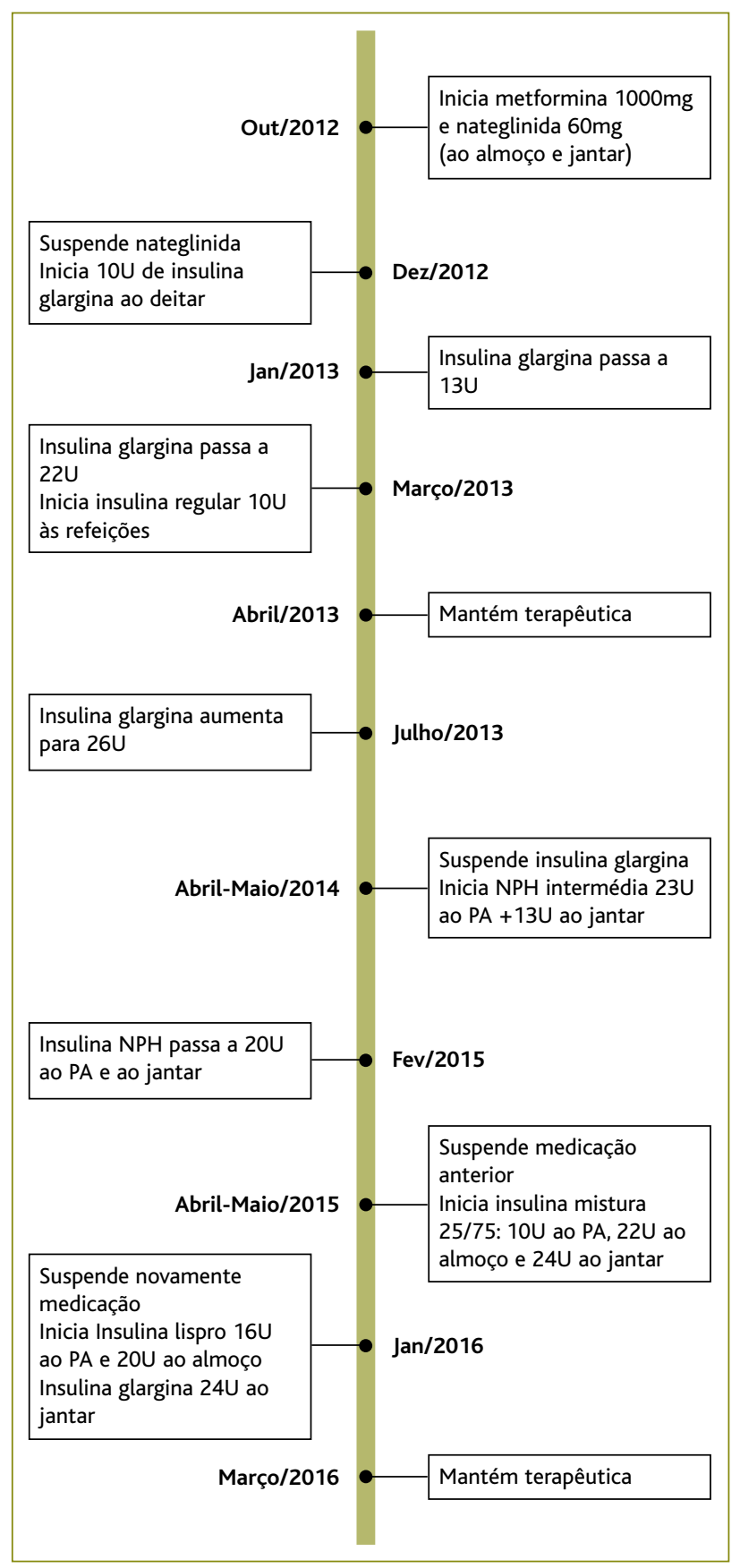

Figura 1. Resumo da evolução da doença ao longo do tempo. Legenda: APDP = Associação Protetora dos Diabéticos de Portugal; DM1 = diabetes mellitus tipo 1; Glicemia p.p. = glicemia pós-prandial; LADA = diabetes latente autoimune do adulto.

che da manhã. Em janeiro de 2016 é internado por cetoacidose diabética. Nessa altura apresenta HbAlc 12,7\% pelo que é novamente ajustada a insulina com os se-

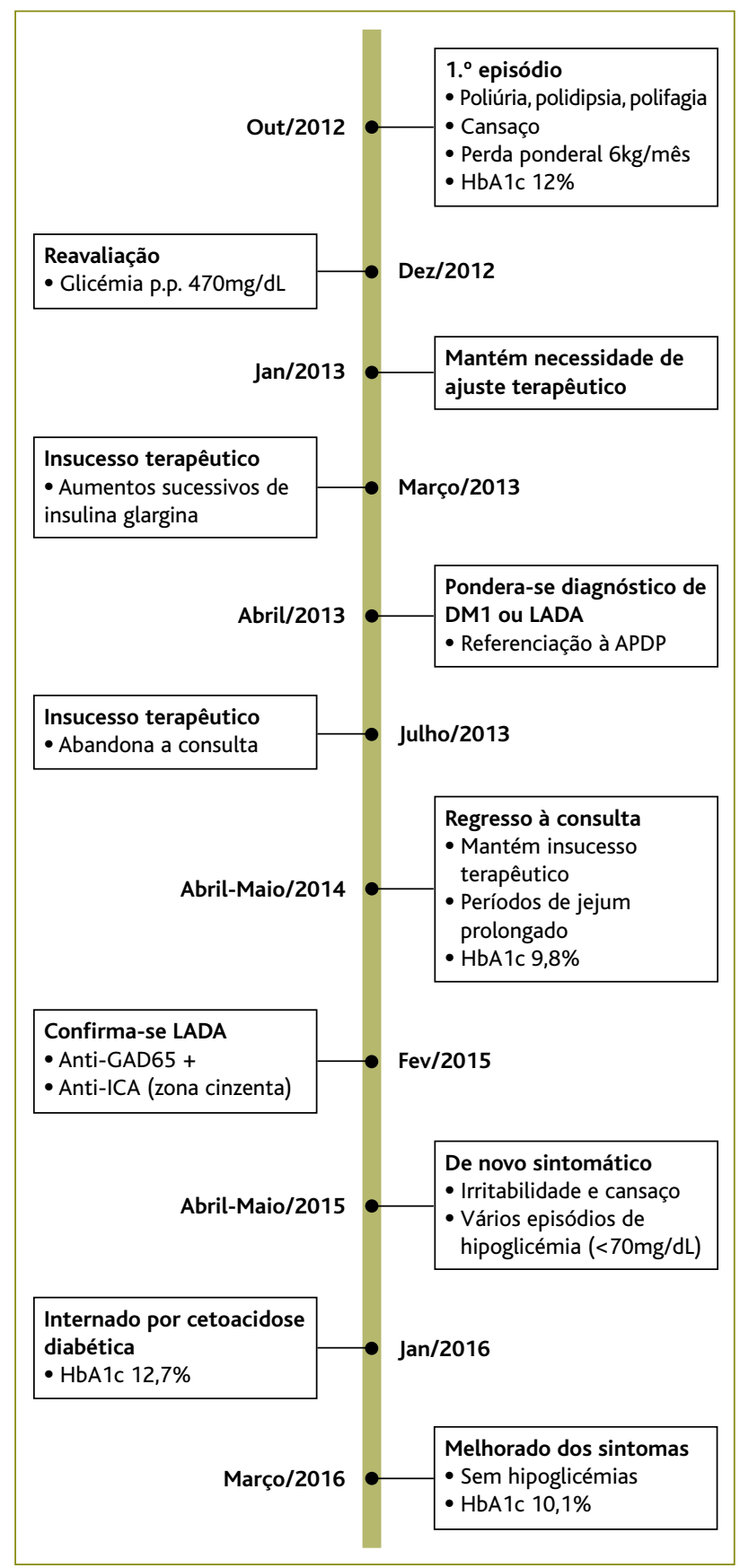

Figura 2. Resumo da evolução terapêutica ao longo do tempo.

guintes tipos e doses: insulina lispro $16 \mathrm{U}$ ao pequeno-almoço e $20 \mathrm{U}$ ao almoço; insulina basal $24 \mathrm{U}$ ao jantar. $\mathrm{Na}$ última avaliação, em março de 2016, apresenta uma melhoria do cansaço, sem registo de novos episódios de hipoglicémia e analiticamente uma HbAlc 10,1\%. 


\section{COMENTÁRIO}

O caso clínico que se descreve releva a influência das condições socioeconómicas no diagnóstico e gestão da diabetes autoimune latente do adulto (LADA).

No caso deste doente em particular, os antecedentes familiares apontam para uma diabetes de origem autoimune, dada a idade de diagnóstico do pai. O contexto sócioeconómico e os seus antecedentes pessoais condicionaram hábitos alimentares desadequados, com refeições escassas e em períodos irregulares, ricas em hidratos de carbono, uma vez que estes eram os alimentos de mais fácil acesso, além de má adesão aos cuidados de saúde, nomeadamente na frequência de consultas médicas, de enfermagem e adesão à terapêutica. Estas condições escondem o verdadeiro insucesso dos fármacos no controlo da doença, dificultando o tratamento e até o próprio diagnóstico da mesma. Por outro lado, permanece a necessidade de introduzir insulina na terapêutica dos doentes com LADA, que algumas vezes é protelada por suspeita de incumprimento terapêutico e maus hábitos alimentares. ${ }^{5}$

A alimentação de um doente diabético deve centrar-se em produtos com poucos hidratos de carbono e pobres em calorias. O objetivo relativamente aos hidratos de carbono centra-se na evicção de picos hiperglicémicos pós-prandiais, que são influenciados quer pela quantidade, quer pelo tipo de hidratos de carbono ingeridos. ${ }^{10}$

Estudos recentes transversais parecem apontar para um benefício na introdução de inibidores da DPP4 na terapêutica dos doentes com LADA. Se, por um lado, estudos apontam para uma maior atividade da DPP4 em doentes com LADA quando comparados com diabéticos tipo 1 e 2, por outro, estes fármacos têm efeito não só no controlo metabólico, como também parecem intervir na história natural da doença, melhorando a função residual das células $\beta \cdot{ }^{6,9}$ Estes benefícios parecem resultar do efeito sinérgico dos inibidores da DPP4 com a utilização de insulina, que ocorrem de forma independente aos mecanismos de origem no sistema imune, levando indiretamente à redução do stress das células $\beta$, redução do glucagon e regeneração das células b. Além do mais, os doentes com LADA apresentam um pior controlo glicémico, pelo que se pode concluir que a insulina, ainda que seja a primeira linha de tratamento, pode ter maior benefício quando não utilizada de forma isolada. ${ }^{6,8}$
Por estes motivos, e ainda que careça de estudos com maior robustez, este doente poderá vir a beneficiar de uma terapêutica em que se inclua um iDPP4, numa perspetiva de alteração da história natural da doença, neste caso, a LADA.

Apesar de haver momentos em que o doente se sentia melhor e por isso faltava ao seguimento em consulta, a proximidade dos cuidados e a facilidade de agendamento permitiram que fosse sempre restabelecido este seguimento, contribuindo para que, apesar de tudo, o diagnóstico fosse mais célere.

A má adesão à terapêutica e o absentismo às consultas tornaram mais difíceis o controlo da doença e a gestão da terapêutica num doente com contexto social por si já muito desfavorável. Este aspeto relembra a importância da participação ativa dos familiares mais próximos, que devem estar conscientes da doença do familiar, conhecer o tratamento e as correções alimentares que devem seguir e quais as metas a atingir, funcionando igualmente como ponto de contacto e de apoio. Neste caso em particular, a relação conflituosa do doente com a irmã foi mais um elemento perturbador para o sucesso terapêutico. Por forma a minimizar o impacto do contexto sócioeconómico, e uma vez que o médico de família trabalha diretamente junto da comunidade, foi possível mobilizar recursos com o objetivo de assegurar cuidados alimentares mais adequados a este utente.

Importa, ainda, não descurar a necessidade de capacitar o doente pelo seu próprio tratamento, em especial em casos de doença crónica, como a diabetes autoimune latente do adulto. Entende-se que, além de assegurar o cumprimento da terapêutica, é fundamental assegurar hábitos de alimentação saudável, fornecendo ferramentas personalizadas adequadas às necessidades específicas do doente em questão.

Em conclusão, salienta-se que cabe ao médico de família, como gestor do doente, integrar todos os problemas do mesmo, promover bons hábitos alimentares e apoiar a adaptação à doença crónica.

\section{REFERÊNCIAS BIBLIOGRÁFICAS}

1. Brahmkshatriya PP, Mehta AA, Saboo BD, Goyal RK. Characteristics and prevalence of latent autoimmune diabetes in adults (LADA). ISRN Pharmacol. 2012;2012:580202.

2. Poudel RR. Latent autoimmune diabetes of adults: from oral hypogly- 
cemic agents to early insulin. Indian J Endocrinol Metab. 2012;16 Suppl 1:S41-6.

3. Pollak F, Vásquez F. Diabetes autoinmune (latente) del adulto [Latent autoimmune diabetes in adults]. Rev Med Chile. 2012;140(11):147681. Spanish

4. Yousefzadeh G, Gozashti M, Najafipour H, Gholamhosseinian NA, Bahramnejad A, Shokouhi M. Common autoimmune biomarkers, thyroid hormonal abnormalities, and beta cells dysfunction in patients with latent autoimmune diabetes in adults with type II diabetes mellitus. Diabetes Metab Syndr. 2016;10(1 Suppl 1):S52-5.

5. O'Neal KS, Johnson JL, Panak RL. Recognizing and appropriately treating latent autoimmune diabetes in adults. Diabetes Spectr. 2016;29(4):249-52.

6. Duvnjak L, Blaslov K, Vučić Lovrenčić M, Knežević Ćuća J. Persons with latent autoimmune diabetes in adults express higher dipeptidyl peptidase- 4 activity compared to persons with type 2 and type 1 diabetes. Diabetes Res Clin Pract. 2016;121:119-26.

7. Stenström G, Gottsäter A, Bakhtadze E, Berger B, Sundkvist G. Latent autoimmune diabetes in adults: definition, prevalence, beta-cell function, and treatment. Diabetes. 2005;54 Suppl 2:S68-72.
8. Laugesen E, Østergaard JA, Leslie RD. Latent autoimmune diabetes of the adult: current knowledge and uncertainty. Diabet Med. 2015;32(7):843-52.

9. Zhao Y, Yang L, Xiang Y, Liu L, Huang G, Long Z, et al. Dipeptidyl peptidase 4 inhibitor sitagliptin maintains $\beta$-cell function in patients with recent-onset latent autoimmune diabetes in adults: one year prospective study. J Clin Endocrinol Metab. 2014;99(5):E876-80.

10. Khazrai YM, Defeudis G, Pozzilli P. Effect of diet on type 2 diabetes mellitus: a review. Diabetes Metab Res Rev. 2014;30 Suppl 1:24-33.

\section{CONFLITO DE INTERESSES}

Os autores declaram não ter quaisquer conflitos de interesse.

\author{
ENDEREÇO PARA CORRESPONDÊNCIA \\ Ana Rita Mendes \\ E-mail: anarita_m@msn.com \\ https://orcid.org/0000-0003-2381-7876
}

Recebido em 13-04-2017

Aceite para publicação em 04-06-2019

\section{ABSTRACT}

\section{A CASE OF DIABETES HARD TO CONTROL}

Introduction: Latent autoimmune diabetes of the adult (LADA) is characterized by the presence of diabetes mellitus in individuals under 50 years old, with initial polyphagia, polydipsia and/or polyuria, BMI $<25 \mathrm{~kg} / \mathrm{m} 2$ and a personal or family history of autoimmune diseases.

Case report: Male, 46 years old, male, unemployed, with a personal history of cognitive impairment, toxic substance abuse, and depressive and anxiety disorders. He has a family history of diabetes mellitus diagnosed to his father at 40 -years old. He also has a conflicted relationship with his sister. On October 2012, presented with fatigue, xerostomia, dry mouth, hyperphagia, polydipsia, polyuria and weight loss with new episodes of alcohol and tobacco abuse, and inappropriate behaviour. We hypothesize this was an inaugural diabetes mellitus condition, with an $\mathrm{HbA} 1 \mathrm{c}$ of $12 \%$ and fasting glucose of $254 \mathrm{mg} / \mathrm{dL}$. He started treatment with metformin and nateglinide. However, due to inappropriate control of the disease, we had to intensify therapeutic gradually, starting insulin administration two months after diagnosis. He was referral to a diabetology appointment, but he missed for several times both their and our appointments until he was diagnosed with latent autoimmune diabetes of the adult (LADA), on February 2015, when he had positive anti-GAD65 antibodies. Due to extreme poverty he missed many meals suffering some episodes of hypoglycaemia. By that time, he felt tired and irritable. On January 2016 he was admitted in the emergency room because of diabetic ketoacidosis. Since that episode, his social support improved, he had no further symptoms of hypoglycaemia, although remains with inadequate levels of $\mathrm{HbA} 1 \mathrm{c}$.

Comment: This case report shows the impact of social-economic conditions, personal history and familiar relationships on the treatment of chronic diseases like LADA. Inadequate eating habits, with scarce and irregular meals and poor adherence to health care and therapy, have led to ineffective disease control. With this case report, we want to highlight the importance of hold the patient responsible for his treatment, as well as involving his closest family. In this particular case, the conflictual relationship with his sister proves to be a disturbing factor to achieve a successful therapy accomplishment.

Keywords: Diabetes; Autoimmune; Diet; Socioeconomic factors. 